Accepted for publication in Phys. Rev. E

\title{
Interaction and flocculation of spherical colloids wetted by a surface-induced corona of paranematic order
}

\author{
Paolo Galatola* \\ LBHP (UMR 7057), Université Paris 7-Denis Diderot and FR CNRS 2438 "Matière et Systèmes Complexes", \\ Case 7056, 2 place Jussieu, F-75251 Paris cedex 05, France \\ Jean-Baptiste Fournien $\dagger$ \\ Laboratoire de Physico-Chimie Théorique and FR CNRS 2438 "Matière et Systèmes Complexes", \\ ESPCI, 10 rue Vauquelin, F-75231 Paris cedex 05, France \\ Holger Stark \\ Universität Konstanz, Fachbereich Physik, D-78457 Konstanz, Germany
}

(Dated: October 31, 2018)

\begin{abstract}
Particles dispersed in a liquid crystal above the nematic-isotropic phase transition are wetted by a surface-induced corona of paranematic order. Such coronas give rise to pronounced twoparticle interactions. In this article, we report details on the analytical and numerical study of these interactions published recently [Phys. Rev. Lett. 86, 3915 (2001)]. We especially demonstrate how for large particle separations the asymptotic form of a Yukawa potential arises. We show that the Yukawa potential is a surprisingly good description for the two-particle interactions down to distances of the order of the nematic coherence length. Based on this fact, we extend earlier studies on a temperature induced flocculation transition in electrostatically stabilized colloidal dispersions [Phys. Rev. E 61, 2831 (2000)]. We employ the Yukawa potential to establish a flocculation diagram for a much larger range of the electrostatic parameters, namely the surface charge density and the Debye screening length. As a new feature, a kinetically stabilized dispersion close to the nematicisotropic phase transition is found.
\end{abstract}

PACS numbers: 82.70.Dd, 61.30.Cz, 61.30.Hn

\section{INTRODUCTION}

Colloidal dispersions are suspensions in a host fluid of solid or liquid particles, of radius ranging from $10 \mathrm{~nm}$ to $10 \mu \mathrm{m} \mathrm{[1].} \mathrm{They} \mathrm{are} \mathrm{long-lived} \mathrm{metastable} \mathrm{states} \mathrm{of}$ matter that present great interest both from a fundamental point of view and in applications, as, e.g., in paints, coatings, foods and drugs. Their stability against flocculation is a key issue in colloidal physics, since the properties of a colloidal dispersion drastically change when a transition from a dispersed to an aggregate state occurs: to prevent coagulation due to attractive van der Waals forces, colloidal particles are usually treated in order to produce electrostatic or steric repulsive interactions.

Recently, great attention was paid to liquid crystal colloidal suspensions and emulsions, i.e., dispersions of solid particles or liquid droplets - respectively - in a liquid crystal 2, 3, 4, 5, 6]. In the case of emulsions in a nematic phase, the radial anchoring of the nematic molecules at the surface of the droplets yields topological defects of the nematic texture in the vicinity of the droplets. These defects produce strong repulsions that stabilize the liquid droplets against coalescence [4]. Simi-

\footnotetext{
*Electronic address: galatola@ccr.jussieu.fr

${ }^{\dagger}$ Electronic address: jbf@turner.pct.espci.fr

${ }^{\ddagger}$ Electronic address: holger.stark@uni-konstanz.de
}

lar effects are present also in other anisotropic fluid hosts, such as lyotropic solutions of anisotropic micelles 7], and in different liquid crystal phases, as in cholesterics [8]. Such systems form composite materials with unusual properties 9, 10, 11].

Even more recently, there has been a growing interest in the pretransitional surface-induced interactions mediated by the paranematic order arising in the vicinity of surfaces at temperatures above the nematic-isotropic phase transition. In [12], the force between two parallel plates immersed in a liquid crystal slightly above the bulk isotropic-nematic transition was theoretically investigated. In 13], the force between a flat surface and a glass microsphere, mediated by the surface-induced paranematic order, was experimentally measured using an atomic force microscope. The measurements were interpreted in terms of the force between two flat surfaces using the Derjaguin approximation [14].

Interesting effects regarding the stabilization of colloids are thus to be expected in the isotropic phase of a nematogenic material when the colloids are wetted by a corona of paranematic order [15, 16, 17]. Two effects compete: an attraction due to the favorable overlapping of the paranematic halos (which reduces the volume of the thermodynamically unvaforable paranematic phase) and a repulsion due to the distortion of the director field. The vicinity of a phase transition may give a critical character to the stabilization mechanism and yield rich reversible phase-separation behaviors, as pre- 
dicted in 18, 19] for a simpler system with a scalar orderparameter. In Ref. 15], the interaction of two spherical particles immersed in a liquid crystal above the nematicisotropic phase transition was investigated using a uniaxial ansatz function for the paranematic order, in the limit of sphere radii large with respect to the nematic coherence length. This approximate solution was used in Ref. 20] to analyze the stability of a suspension of such colloidal particles, in the presence of destabilizing van der Waals attractive interactions and stabilizing electrostatic repulsions. In Ref. [16], an exact numerical solution for the above paranematic interaction was obtained, using a multipolar expansion of the tensor order-parameter and taking into account biaxiality. The possibility of stabilizing colloidal particles was discussed, emphasizing the case of rather small particles, i.e., of size comparable to several nematic coherence lengths. A large-separation asymptotic analytic form of Yukawa type was also obtained, that was shown to very closely describe the exact interaction between large particles up to separations comparable to the nematic coherence length [21]. Alternative derivations of the Yukawa potential were given in Ref. 17] based on a Debye-Hückel type approximation and a geometric view.

In this work, we revise in detail the solution found in Ref. 16], which is exact in the limit of weak surfaceinduced paranematic order. We give a particular emphasis on the meaning and limits of validity of the approximate asymptotic solution. The latter is used to analyze in detail the stability of a colloidal suspension in the presence of van der Waals and electrostatic interactions. We obtain and discuss general diagrams for the stability of the suspension as a function of the various relevant parameters.

The detailed plan of our paper is the following. In Section [11 we describe our Landau-de Gennes [22] model for the bulk and the surface free-energy. In Section III we present the corresponding equilibrium equations and formulate the general multipolar expansion for the bulk order-parameter in spherical coordinates. This expansion is used in Section IV to obtain the exact solution for an isolated spherical particle. The interaction of two such particles is discussed in Section $\square$ In particular, in Section $\mathrm{VB} 1$ we derive the asymptotic interaction energy. The asymptotic solution is analyzed in Section VB2 in terms of the superposition of single-particle solutions. In Section $\mathrm{VB} 3$ we discuss the limits of validity of the Derjaguin approximation. In Section $\overline{\nabla C}$ we present an exact numerical solution for the interaction of two particles. The comparison between the asymptotic interaction energy and the exact numerical one is performed in Section $\nabla C 1$ In Section $\nabla C 2$ we discuss the texture between two interacting particles, and in Section $\nabla C 3$ we analyze the defect ring that appears in the paranematic texture between the two particles. Finally, in Section VI] using our asymptotic solution, we discuss the stability of such colloidal dispersions.

\section{MODEL}

Let us first describe the nematogenic phase in which the colloids will be placed. Nematic liquid crystals are anisotropic liquid phases, in which elongated molecules display a long-range orientational order. This order is described by a symmetric traceless tensorial orderparameter $Q_{i j}(i, j=1,2,3)$, since nematics are nonpolar [22]. The eigenvectors of $Q_{i j}$ represent the axes of main molecular orientation and its eigenvalues describe the amount of orientational ordering in each direction. Usually, nematics are uniaxial phases, however biaxiality naturally arises in inhomogeneous situations, e.g., in the vicinity of defects 23]. The Landau-de Gennes expansion of the bulk free-energy density [22] has the form [24, 25]

$$
\begin{aligned}
f_{b}= & \frac{a}{2} Q_{i j} Q_{i j}-\frac{b}{3} Q_{i j} Q_{j k} Q_{k i}+\frac{c}{4}\left(Q_{i j} Q_{i j}\right)^{2} \\
& +\frac{L}{2} Q_{i j, k} Q_{i j, k} .
\end{aligned}
$$

Summation over repeated indices is implied and comma indicates derivation. The coefficients are such that $a=$ $\alpha\left(T-T^{*}\right)$, with $\alpha, c$, and $L$ positive. The temperature $T^{*}$ is the lowest temperature $T$ at which the isotropic phase can exist. The presence of the cubic term $Q_{i j} Q_{j k} Q_{k l}$ in the homogeneous part implies that the nematic-isotropic transition is first-order. To simplify the description, we have introduced only one gradient term in Eq. (1), which corresponds to the usual one-constant approximation [26]. The most general expansion of the surface freeenergy density is 27,28 ]

$$
\begin{aligned}
f_{s}= & v_{1} Q_{i j} \nu_{i} \nu_{j}+\frac{v_{21}}{2} Q_{i j} Q_{i j}+\frac{v_{22}}{2} Q_{i j} Q_{j k} \nu_{i} \nu_{k} \\
& +\frac{v_{23}}{2} Q_{i j} Q_{k l} \nu_{i} \nu_{j} \nu_{k} \nu_{l}+\mathcal{O}\left(Q^{3}\right),
\end{aligned}
$$

where the $\nu_{i}$ 's $(i=1,2,3)$ are the components of the outward normal $\boldsymbol{\nu}$ of the surface. Owing to the linear term, the surface always locally favor a nonzero order.

Since we are dealing with the isotropic phase, and, in addition, we assume a weak surface-induced order and temperatures not too close to the phase transition, we can neglect the third and higher-order terms in $f_{b}$ and $f_{s}$ [15, 16]. Then, the free-energy being quadratic, exact calculations are feasible. To simplify, we shall also retain only the simplest quadratic surface term, by setting $v_{22}=$ 0 and $v_{23}=0$. Then the free-energy densities can be put in the form

$$
\begin{aligned}
f_{b} & =\frac{a}{2} Q_{i j} Q_{i j}+\frac{L}{2} Q_{i j, k} Q_{i j, k} \\
f_{s} & =\frac{1}{2} W\left(Q_{i j}-Q_{i j}^{(0)}\right)\left(Q_{i j}-Q_{i j}^{(0)}\right),
\end{aligned}
$$

where

$$
Q_{i j}^{(0)}=S_{0}\left(\nu_{i} \nu_{j}-\frac{1}{3} \delta_{i j}\right)
$$


is the preferred order-parameter at the surface. The surface free-energy density (3b) is compatible with the experimentally measured anchoring in the nematic phase [28].

In the following, unless otherwise specified, we shall normalize lengths with respect to the nematic correlation length $\xi=(L / a)^{1 / 2}$ and energies with respect to $F_{0}=a \xi^{3} S_{0}^{2}$. Because of the conditions that $Q_{i j}$ must be symmetric and traceless, the free-energy densities (3) are not diagonal in the five independent components of $Q_{i j}$. Defining the following set of components:

$$
\begin{aligned}
& q_{0}=\frac{Q_{x x}-Q_{y y}}{S_{0}}, \\
& q_{1}=\frac{Q_{y z}}{S_{0}}=\frac{Q_{z y}}{S_{0}}, \\
& q_{2}=\frac{Q_{x z}}{S_{0}}=\frac{Q_{z x}}{S_{0}}, \\
& q_{3}=\frac{Q_{x y}}{S_{0}}=\frac{Q_{y x}}{S_{0}}, \\
& q_{4}=\frac{Q_{z z}}{S_{0}},
\end{aligned}
$$

the normalized free-energy takes the following diagonal form:

$$
F=\sum_{i=0}^{4} c_{i}\left\{\int\left[q_{i}^{2}+\left(\nabla q_{i}\right)^{2}\right] d^{3} r+w \int\left(q_{i}-q_{i}^{(0)}\right)^{2} d^{2} r\right\}
$$

where $c_{0}=1 / 4, c_{1}=c_{2}=c_{3}=1, c_{4}=3 / 4$, and $w=W / a \xi$ is the normalized anchoring strength. The first integral is over the bulk and the second one over the surface of each colloidal particle. Thanks to this transformation, the tensorial problem associated with the paranematic order between the colloids is reduced to the superposition of five independent scalar problems.

\section{EQUILIBRIUM EQUATIONS AND SOLUTIONS}

At equilibrium, the order-parameter texture minimizes the free-energy (6). By setting to zero the variation $\delta F$ of the free-energy, associated with arbitrary infinitesimal variations of the $q_{i}$ components, we obtain the equilibrium equations

$$
\left(\nabla^{2}-1\right) q_{i}=0
$$

in the bulk, and

$$
\boldsymbol{\nu} \cdot \nabla q_{i}=w\left(q_{i}-q_{i}^{(0)}\right)
$$

on the surface of each colloidal particle. Determining the paranematic order outside the colloidal particles is therefore somewhat similar to solving an electrostatic problem with mixed boundary conditions in which the potential is replaced by the $q_{i}$ fields and the standard Laplacian operator by $\nabla^{2}-1$. We shall therefore use multipolar expansions. Actually, the operator $\nabla^{2}-1$ is the operator associated with a massive boson field, which implies a short-range interaction [29].

\section{A. Multipolar expansion}

In spherical coordinates $(r, \theta, \phi)$, the spherical harmonics $Y_{\ell m}(\theta, \phi)$ (for their definition see Appendix A) are eigenfunctions of the angular part of the Laplacian operator $\nabla^{2}$ and form a complete basis. Then, each of the $q_{i}$ 's can be expanded as

$$
q_{i}(r, \theta, \phi)=\sum_{\ell=0}^{\infty} \sum_{m=-\ell}^{\ell} q_{i}^{\ell m} u_{\ell}(r) Y_{\ell m}(\theta, \phi)
$$

where the functions $u_{\ell}(r)$ obey the equations

$$
\frac{d}{d r}\left(r^{2} \frac{d u_{\ell}}{d r}\right)=\left[r^{2}+\ell(\ell+1)\right] u_{\ell},
$$

which follows by inserting expansion (9) into the bulk equation (7). The solution of Eq. (10) that is regular at infinity can be expressed as

$$
u_{\ell}(r)=\sqrt{\frac{2}{\pi r}} K_{\ell+1 / 2}(r)
$$

where the $K_{\ell+1 / 2}(r)$ are modified Bessel functions of halfinteger order. Explicitly,

$$
\begin{aligned}
u_{0}(r) & =\frac{e^{-r}}{r} \\
u_{1}(r) & =\frac{e^{-r}}{r}\left(1+\frac{1}{r}\right), \\
u_{2}(r) & =\frac{e^{-r}}{r}\left(1+\frac{3}{r}+\frac{3}{r^{2}}\right), \\
u_{\ell+1}(r) & =\frac{2 \ell+1}{r} u_{\ell}(r)+u_{\ell-1}(r) \text { for } \ell \geq 1 .
\end{aligned}
$$

With such an expansion, the paranematic order is thus completely described by the set of coefficients $q_{i}^{\ell m}$.

\section{B. Equilibrium free-energy}

By integrating by parts Eq. (6) and using the equilibrium equations (7) and (8), the normalized freeenergy (6) associated with an equilibrium solution can be written as

$$
F=w \sum_{i=0}^{4} c_{i} \int q_{i}^{(0)}\left(q_{i}^{(0)}-q_{i}\right) d^{2} r
$$

where the integrations run over the surfaces of all the particles. 


\section{PARANEMATIC ORDER AROUND ONE PARTICLE}

Let us consider a spherical particle of reduced radius $R$. In spherical coordinates $(r, \theta, \phi)$, the normal to the surface of the particle is given by $\boldsymbol{\nu}=\sin \theta \cos \phi \hat{\mathbf{x}}+$ $\sin \theta \sin \phi \hat{\mathbf{y}}+\cos \theta \hat{\mathbf{z}}$. Therefore, the preferred surface order-parameter (44) has the following $q$-components [see Eqs. (5)]

$$
\begin{aligned}
& q_{0}^{(0)}=\sin ^{2} \theta \cos 2 \phi=4 \sqrt{\frac{2 \pi}{15}} Y_{22}^{R}(\theta, \phi) \\
& q_{1}^{(0)}=\frac{1}{2} \sin 2 \theta \sin \phi=-2 \sqrt{\frac{2 \pi}{15}} Y_{21}^{I}(\theta, \phi) \\
& q_{2}^{(0)}=\frac{1}{2} \sin 2 \theta \cos \phi=-2 \sqrt{\frac{2 \pi}{15}} Y_{21}^{R}(\theta, \phi) \\
& q_{3}^{(0)}=\frac{1}{2} \sin ^{2} \theta \sin 2 \phi=2 \sqrt{\frac{2 \pi}{15}} Y_{22}^{I}(\theta, \phi) \\
& q_{4}^{(0)}=\cos ^{2} \theta-\frac{1}{3}=\frac{4}{3} \sqrt{\frac{\pi}{5}} Y_{20}(\theta, \phi),
\end{aligned}
$$

where

$$
\begin{aligned}
Y_{\ell m}^{R} & =\frac{1}{2}\left(Y_{\ell m}+Y_{\ell m}^{*}\right), \\
Y_{\ell m}^{I} & =\frac{1}{2 i}\left(Y_{\ell m}-Y_{\ell m}^{*}\right)
\end{aligned}
$$

are the real and imaginary part, respectively, of the spherical harmonics. The boundary conditions (8) become simply

$$
\left.\frac{\partial q_{i}}{\partial r}\right|_{r=R}=w\left[q_{i}(R, \theta, \phi)-q_{i}^{(0)}(\theta, \phi)\right] .
$$

Replacing in Eq. (16) $q_{i}$ by its multipolar expansion (9) and $q_{i}^{(0)}$ by its expression given in Eqs. (14), and identifying the coefficients of the spherical harmonics, yields the solution

$$
\left(\begin{array}{c}
q_{0} \\
q_{1} \\
q_{2} \\
q_{3} \\
q_{4}
\end{array}\right)=\sqrt{\frac{2 \pi}{15}}\left(\begin{array}{c}
2 Y_{22}^{R}(\theta, \phi) \\
-Y_{21}^{I}(\theta, \phi) \\
-Y_{21}^{R}(\theta, \phi) \\
Y_{22}^{I}(\theta, \phi) \\
\sqrt{\frac{2}{3}} Y_{20}(\theta, \phi)
\end{array}\right) 2 \mathcal{A}(R, w) e^{R} u_{2}(r)
$$

where the amplitude $\mathcal{A}(R, w)$ is given by

$$
\mathcal{A}=\frac{R^{4} w}{R^{3}(w+1)+R^{2}(3 w+4)+3 R(w+3)+9}
$$

and $u_{2}(r)$ is given by Eq. (12c).

Since Eq. (17) has the same angular structure as Eqs. (14), the order-parameter is uniaxial with a radial director $\mathbf{n}=\boldsymbol{\nu}$, i.e., $Q_{i j}=S(r)\left(\nu_{i} \nu_{j}-\frac{1}{3} \delta_{i j}\right)$, where

$$
S(r)=S_{0} \mathcal{A}(R, w)\left(1+\frac{3}{r}+\frac{3}{r^{2}}\right) \frac{e^{R-r}}{r}
$$

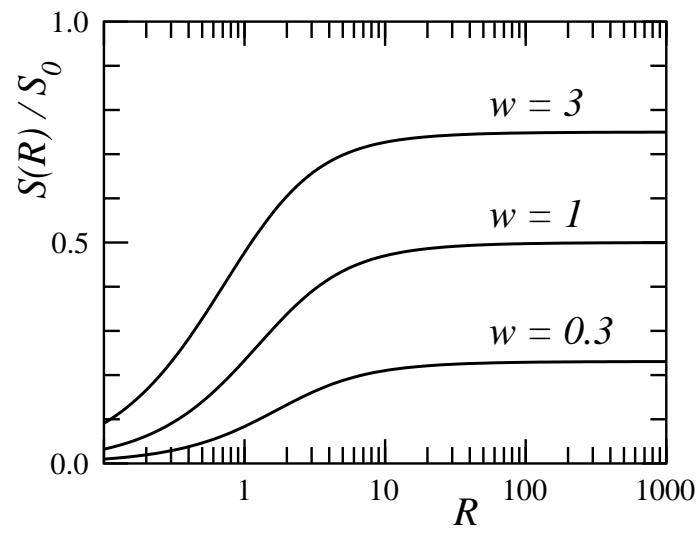

FIG. 1: Normalized scalar order-parameter $S(R) / S_{0}$ at the surface of one isolated spherical colloid as a function of its normalized radius $R$ for various reduced anchoring strength $w$.

is the uniaxial scalar order-parameter. Note that whatever the size of the particle, the paranematic order relaxes on the coherence length $\xi$ (equal to unity in reduced units). The behavior of the scalar order-parameter at the surface of the particle $(r=R)$, as a function of the radius $R$, is shown in Fig. 11 For large values of $R$, the surface order tends to $S(R) \simeq S_{0} w /(1+w)$ which corresponds to the limit of a planar interface. For $R \lesssim 10$ (in units of $\xi$ ), the surface order-parameter is reduced because of the energy cost associated with the splay of the director.

The free-energy of the particle is obtained from Eq. (13). With the help of Eqs. (14) and (17) and the orthonormality of the spherical harmonics, we find

$$
F_{1}=\frac{4 \pi}{3} \mathcal{A}(R, w)\left(R+4+\frac{9}{R}+\frac{9}{R^{2}}\right) .
$$

\section{INTERACTION OF TWO PARTICLES}

Let us now consider two identical spherical particles of reduced radius $R$ separated by a center-to-center distance $d$. We introduce three spherical coordinate systems: a global one, $(r, \theta, \phi)$, symmetrically placed with respect to the particles, and two local ones, $\left(r_{1}, \theta_{1}, \phi\right)$ and $\left(r_{2}, \theta_{2}, \phi\right)$, centered on the two particles, as indicated in Fig. 2]

\section{A. General formalism}

Using the symmetry of the system allows to reduce the number of free parameters in the tensorial orderparameter field. For a given point $P$, the plane $\Pi_{P}$ passing through $P$ and the $z$-axis is a symmetry plane. Thus, the frame $\left(\mathbf{e}^{(1)}, \mathbf{e}^{(2)}, \mathbf{e}^{(3)}\right)$ in which $Q_{i j}$ is diagonal has two directions $\mathbf{e}^{(1)}$ and $\mathbf{e}^{(2)}$ in the plane $\Pi_{P}$ and the third, $\mathbf{e}^{(3)}$, normal to it. This frame can be parametrized by 


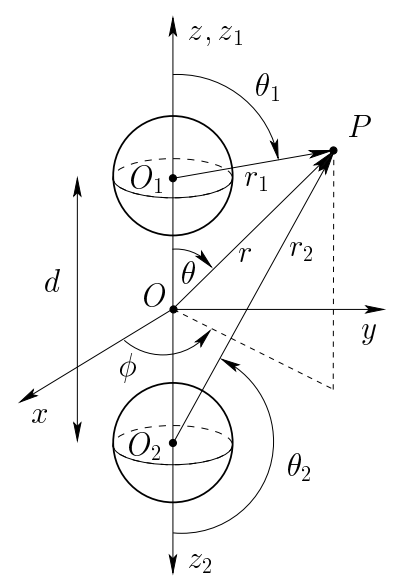

FIG. 2: Geometry for the calculation of the paranematic interaction between two identical spherical colloidal particles separated by a center-to-center distance $d$. The origin of the $z$-axis is in the midpoint $O$ between the centers of the two particles, that of $z_{1}$ in the center $O_{1}$ of the upper particle, and that of $z_{2}$ in the center $\mathrm{O}_{2}$ of the lower one.

the angle $\Theta(r, \theta)$ that $\mathbf{e}^{(1)}$ makes with the $z$-axis:

$$
\begin{aligned}
& \mathbf{e}^{(1)}=\sin \Theta \cos \phi \hat{\mathbf{x}}+\sin \Theta \sin \phi \hat{\mathbf{y}}+\cos \Theta \hat{\mathbf{z}} \\
& \mathbf{e}^{(2)}=\cos \Theta \cos \phi \hat{\mathbf{x}}+\cos \Theta \sin \phi \hat{\mathbf{y}}-\sin \Theta \hat{\mathbf{z}}, \\
& \mathbf{e}^{(3)}=-\sin \phi \hat{\mathbf{x}}+\cos \phi \hat{\mathbf{y}}
\end{aligned}
$$

Therefore, the order-parameter tensor $Q_{i j}$ can be written as

$$
Q_{i j}=\lambda_{1} e_{i}^{(1)} e_{j}^{(1)}+\lambda_{2} e_{i}^{(2)} e_{j}^{(2)}+\lambda_{3} e_{i}^{(3)} e_{j}^{(3)},
$$

where its eigenvalues $\lambda_{i}$ satisfy

$$
\lambda_{3}=-\left(\lambda_{1}+\lambda_{2}\right),
$$

since $Q_{i j}$ is traceless. The corresponding $q$ components (5) are therefore

$$
\begin{aligned}
q_{0} & =\alpha(r, \theta) \cos 2 \phi, \\
q_{1} & =\beta(r, \theta) \sin \phi, \\
q_{2} & =\beta(r, \theta) \cos \phi, \\
q_{3} & =\frac{1}{2} \alpha(r, \theta) \sin 2 \phi, \\
q_{4} & =\gamma(r, \theta),
\end{aligned}
$$

with

$$
\begin{aligned}
& \alpha(r, \theta)=\frac{\lambda_{1}}{S_{0}}\left(1+\sin ^{2} \Theta\right)+\frac{\lambda_{2}}{S_{0}}\left(1+\cos ^{2} \Theta\right), \\
& \beta(r, \theta)=\frac{\lambda_{1}-\lambda_{2}}{2 S_{0}} \sin 2 \Theta \\
& \gamma(r, \theta)=\frac{\lambda_{1}}{S_{0}} \cos ^{2} \Theta+\frac{\lambda_{2}}{S_{0}} \sin ^{2} \Theta .
\end{aligned}
$$

Hence the order-parameter field around two particles is fully described by the three fields $\alpha(r, \theta), \beta(r, \theta)$, and $\gamma(r, \theta)$.

\section{Multipolar development}

For two particles, the most general expression of $Q_{i j}$ in terms of multipoles must be a sum of two multipolar expansions of the kind (9), each one centered on each one of the particles. The reason is the following. Since a multipolar expansion such as (9) is singular at the origin $(r=0)$, the latter must be put inside one of the particles. Moreover, using only one expansion, one would impose that the analytical continuation of the texture is regular inside the other particle (because the multipolar expansion is regular everywhere but at $r=0$ ), which is not required. Therefore, in the most general case, there must be a second multipolar source inside the other particle. For two identical particles, this is also obvious from symmetry considerations.

Taking into account the general form (24) and the fact that $(x, y)$ is a symmetry plane, the double multipolar expansion takes the form (see also Appendix $\mathbf{A}$ ):

$$
\begin{aligned}
& \alpha(r, \theta)=\sum_{\ell=2}^{\infty} \sum_{p=1}^{2} \alpha_{\ell} u_{\ell}\left(r_{p}\right) P_{\ell}^{2}\left(\cos \theta_{p}\right), \\
& \beta(r, \theta)=\sum_{\ell=1}^{\infty} \sum_{p=1}^{2}(-1)^{p} \beta_{\ell} u_{\ell}\left(r_{p}\right) P_{\ell}{ }^{1}\left(\cos \theta_{p}\right), \\
& \gamma(r, \theta)=\sum_{\ell=0}^{\infty} \sum_{p=1}^{2} \gamma_{\ell} u_{\ell}\left(r_{p}\right) P_{\ell}^{0}\left(\cos \theta_{p}\right) .
\end{aligned}
$$

Thus, at this point, the whole order-parameter texture is uniquely determined by the set of coefficients $\alpha_{\ell}$ (with $\ell=2,3, \ldots), \beta_{\ell}($ with $\ell=1,2, \ldots)$, and $\gamma_{\ell}$ (with $\ell=$ $0,1, \ldots)$.

\section{Boundary conditions}

Because of the presence of the $(x, y)$ symmetry plane, we need to impose the boundary conditions (8) only on one of the particles, let say particle 1. These boundary conditions on the $q$-components transform to boundary conditions on the fields $\alpha, \beta$ and $\gamma$ [see Eqs. (24)], which in the coordinates $\left(r_{1}, \theta_{1}\right)$ relative to particle 1 , take the form

$$
\begin{aligned}
& \left.\frac{\partial \alpha}{\partial r_{1}}\right|_{r_{1}=R}=w\left[\alpha\left(r_{1}=R, \theta_{1}\right)-\frac{1}{3} P_{2}^{2}\left(\cos \theta_{1}\right)\right], \\
& \left.\frac{\partial \beta}{\partial r_{1}}\right|_{r_{1}=R}=w\left[\beta\left(r_{1}=R, \theta_{1}\right)+\frac{1}{3} P_{2}^{1}\left(\cos \theta_{1}\right)\right], \\
& \left.\frac{\partial \gamma}{\partial r_{1}}\right|_{r_{1}=R}=w\left[\gamma\left(r_{1}=R, \theta_{1}\right)-\frac{2}{3} P_{2}^{0}\left(\cos \theta_{1}\right)\right] .
\end{aligned}
$$

Note that these equations are decoupled; therefore the three fields $\alpha, \beta$ and $\gamma$ can be treated independently. 


\section{Coordinate transformation}

To impose the boundary conditions (27) to the multipolar expansions (26), we need to express the spherical coordinates $r_{2}$ and $\theta_{2}$ in terms of $r_{1}$ and $\theta_{1}$. From Fig. 2 we easily obtain

$$
\begin{aligned}
r_{2} & =\sqrt{r_{1}^{2}+d^{2}+2 d r_{1} \cos \theta_{1}} \\
\cos \theta_{2} & =-\frac{r_{1} \cos \theta_{1}+d}{\sqrt{r_{1}^{2}+d^{2}+2 d r_{1} \cos \theta_{1}}} .
\end{aligned}
$$

\section{B. Asymptotic solution}

The exact two-particle order-parameter texture (26) satisfying the boundary conditions (27) cannot be deter- mined analytically, owing to the intricate relations (28) that link the local coordinate systems centered on the two particles. However, in the limit $d \gg 1$, i.e., for distances large with respect to the nematic coherence length $\xi$ (even if $d-2 R \ll R$ ), we can obtain an asymptotic solution by expanding the unknown multipolar coefficients $\alpha_{\ell}, \beta_{\ell}$, and $\gamma_{\ell}$ in series of $e^{-d} / d^{n}$ up to a given order $n$. In the following, we shall obtain the lowest order expansion, $n=1$. It turns out that to this order the only non-zero terms in the multipolar expansions (26) are those up to $\ell=2$.

To begin with, we illustrate the strategy for computing the $\gamma_{\ell}$ coefficients. We start by writing the expansion (26C) on particle $1\left(r_{1}=R\right)$ up to $\ell=2$ :

$$
\begin{aligned}
\gamma\left(r_{1}=R, \theta_{1}\right) & =\gamma_{0}\left[u_{0}(R) P_{0}{ }^{0}\left(\cos \theta_{1}\right)+u_{0}\left(R_{2}\right) P_{0}{ }^{0}\left(\cos \Theta_{2}\right)\right]+\gamma_{1}\left[u_{1}(R) P_{1}^{0}\left(\cos \theta_{1}\right)+u_{1}\left(R_{2}\right) P_{1}^{0}\left(\cos \Theta_{2}\right)\right] \\
& +\gamma_{2}\left[u_{2}(R) P_{2}{ }^{0}\left(\cos \theta_{1}\right)+u_{2}\left(R_{2}\right) P_{2}{ }^{0}\left(\cos \Theta_{2}\right)\right]
\end{aligned}
$$

where $R_{2}=R_{2}\left(\theta_{1}\right)$ is the $r_{2}$ coordinate 28a evaluated on particle $1\left(r_{1}=R\right)$, and, similarly, $\cos \Theta_{2}=$ $\cos \Theta_{2}\left(\theta_{1}\right)$ is the $\cos \theta_{2}$ coordinate (28b) evaluated on particle 1 . Next, we asymptotically develop the functions $u_{n}\left(R_{2}\right) P_{n}{ }^{0}\left(\cos \Theta_{2}\right)$ up to terms of order $e^{-d} / d$ :

$$
u_{n}\left(R_{2}\right) P_{n}^{0}\left(\cos \Theta_{2}\right) \simeq(-1)^{n} \frac{e^{-d}}{d} e^{-R \cos \theta_{1}},
$$

and we redevelop this expansion on the complete basis of Legendre functions of first kind $P_{n}{ }^{0}\left(\cos \theta_{1}\right)$, using the orthogonality relations (A3). Truncating the expansion at $\ell=2$, for the sake of consistency, we arrive at

$$
\begin{aligned}
u_{n}\left(R_{2}\right) P_{n}{ }^{0}\left(\cos \Theta_{2}\right) & \simeq(-1)^{n} \frac{e^{-d}}{d}\left\{\frac{\sinh R}{R} P_{0}{ }^{0}\left(\cos \theta_{1}\right)-3\left[\frac{\cosh R}{R}-\frac{\sinh R}{R^{2}}\right] P_{1}^{0}\left(\cos \theta_{1}\right)\right. \\
& \left.+5\left[\frac{\sinh R}{R}-\frac{3 \cosh R}{R^{2}}+\frac{3 \sinh R}{R^{3}}\right] P_{2}{ }^{0}\left(\cos \theta_{1}\right)\right\} .
\end{aligned}
$$

Replacing Eq. (31) into Eq. (29) yields the required expansion of $\gamma$. To match the boundary conditions, let us determine the corresponding expansion for the radial derivative of $\gamma$. To this aim, it is most efficient to take into account that the radial functions (12) obey the relations

$$
\frac{d u_{n}(r)}{d r}=\frac{n u_{n}(r)}{r}-u_{n+1}(r) .
$$

We then obtain

$$
\begin{aligned}
\left.\frac{\partial \gamma}{\partial r_{1}}\right|_{r_{1}=R} & =\gamma_{0}\left\{-u_{1}(R) P_{0}^{0}\left(\cos \theta_{1}\right)+\frac{\partial}{\partial r_{1}}\left[u_{0}\left(r_{2}\right) P_{0}^{0}\left(\cos \theta_{2}\right)\right]_{r_{1}=R}\right\} \\
& +\gamma_{1}\left\{\left[\frac{u_{1}(R)}{R}-u_{2}(R)\right] P_{1}^{0}\left(\cos \theta_{1}\right)+\frac{\partial}{\partial r_{1}}\left[u_{1}\left(r_{2}\right) P_{1}^{0}\left(\cos \theta_{2}\right)\right]_{r_{1}=R}\right\} \\
& +\gamma_{2}\left\{\left[\frac{2 u_{2}(R)}{R}-u_{3}(R)\right] P_{2}{ }^{0}\left(\cos \theta_{1}\right)+\frac{\partial}{\partial r_{1}}\left[u_{2}\left(r_{2}\right) P_{2}{ }^{0}\left(\cos \theta_{2}\right)\right]_{r_{1}=R}\right\},
\end{aligned}
$$


in which, according to Eq. (31),

$$
\begin{aligned}
\frac{\partial}{\partial r_{1}}\left[u_{n}\left(r_{2}\right) P_{n}{ }^{0}\left(\cos \theta_{2}\right)\right]_{r_{1}=R} & \simeq(-1)^{n} \frac{e^{-d}}{d}\left\{\left[\frac{\cosh R}{R}-\frac{\sinh R}{R^{2}}\right] P_{0}{ }^{0}\left(\cos \theta_{1}\right)\right. \\
& -3\left[\frac{\sinh R}{R}-\frac{2 \cosh R}{R^{2}}+\frac{2 \sinh R}{R^{3}}\right] P_{1}^{0}\left(\cos \theta_{1}\right) \\
& \left.+5\left[\frac{\cosh R}{R}-\frac{4 \sinh R}{R^{2}}+\frac{9 \cosh R}{R^{3}}-\frac{9 \sinh R}{R^{4}}\right] P_{2}{ }^{0}\left(\cos \theta_{1}\right)\right\} .
\end{aligned}
$$

Finally, we insert the relations (29), (31), (33), and (34) in the boundary condition (27c). Because of the orthogonality relations (A3), we get three linear coupled equations for the unknown coefficients $\gamma_{0}, \gamma_{1}$, and $\gamma_{2}$. Solving them to leading order in $e^{-d} / d$ yields, after some lengthy algebra,

$$
\begin{aligned}
& \gamma_{0} \simeq \frac{e^{-d}}{d} \frac{e^{R} R^{4} w\left\{R(w+1)+1-e^{2 R}[R(w-1)+1]\right\}}{3\left[R^{4}(w+1)^{2}+R^{3}\left(3 w^{2}+8 w+5\right)+R^{2}\left(3 w^{2}+15 w+13\right)+6 R(2 w+3)+9\right]}, \\
& \gamma_{1} \simeq \frac{e^{-d}}{d} \frac{e^{R} R^{4} w\left\{R^{2}(w+1)+R(w+2)+2+e^{2 R}\left[R^{2}(w-1)-R(w-2)-2\right]\right\}}{R^{5}(w+1)^{2}+2 R^{4}\left(2 w^{2}+5 w+3\right)+R^{3}\left(6 w^{2}+24 w+19\right)+R^{2}\left(3 w^{2}+30 w+35\right)+3 R(5 w+12)+18}, \\
& \gamma_{2} \simeq \frac{2}{3} \mathcal{A}(R, w) e^{R}\left\{1+\frac{5}{2} \frac{e^{-d}}{d}\left[1-\frac{e^{2 R} \mathcal{A}(R, w)\left[R^{3}(w-1)-R^{2}(3 w-4)+3 R(w-3)+9\right]}{R^{4} w}\right]\right\} .
\end{aligned}
$$

The calculation of the remaining coefficients $\alpha_{\ell}$ and $\beta_{\ell}$ is much simpler since, to leading order in $e^{-d} / d$, they do not depend on $d$, because of the asymptotic behavior

$$
\begin{aligned}
& u_{n}\left(R_{2}\right) P_{n}{ }^{1}\left(\cos \Theta_{2}\right) \propto \frac{e^{-d}}{d^{2}}, \\
& u_{n}\left(R_{2}\right) P_{n}{ }^{2}\left(\cos \Theta_{2}\right) \propto \frac{e^{-d}}{d^{3}} .
\end{aligned}
$$

These coefficients are therefore given by their expressions for an isolated particle

$$
\begin{aligned}
\alpha_{2} & \simeq \frac{1}{3} \mathcal{A}(R, w) e^{R} \\
\beta_{1} & \simeq 0 \\
\beta_{2} & \simeq-\frac{1}{3} \mathcal{A}(R, w) e^{R} .
\end{aligned}
$$

\section{Asymptotic interaction energy}

To calculate the free-energy we use Eq. (13). By symmetry, it is equal to twice the contribution corresponding to the surface of particle 1 . In order to calculate this contribution, we express $\alpha\left(r_{1}=R, \theta_{1}\right), \beta\left(r_{1}=R, \theta_{1}\right)$ and $\gamma\left(r_{1}=R, \theta_{1}\right)$ as an expansion in Legendre functions:

$$
\begin{aligned}
& \alpha\left(r_{1}=R, \theta_{1}\right)=\sum_{n=2}^{\infty} A_{n} P_{n}{ }^{2}\left(\cos \theta_{1}\right), \\
& \beta\left(r_{1}=R, \theta_{1}\right)=\sum_{n=1}^{\infty} B_{n} P_{n}{ }^{1}\left(\cos \theta_{1}\right), \\
& \gamma\left(r_{1}=R, \theta_{1}\right)=\sum_{n=0}^{\infty} C_{n} P_{n}{ }^{0}\left(\cos \theta_{1}\right) .
\end{aligned}
$$

Using Eq. (13) and the orthogonality of the Legendre functions, we obtain

$$
F=\frac{4}{15} \pi R^{2} w\left(10-12 A_{2}+12 B_{2}-3 C_{2}\right) .
$$

The coefficients $A_{2}, B_{2}$ and $C_{2}$ are easily calculated from the asymptotic expansions of $\alpha, \beta$, and $\gamma$ given in the preceding section. We arrive at $F=2 F_{1}+F_{\text {int }}^{\infty}$, where $F_{1}$ is the free-energy (20) of an isolated particle and

$$
\begin{aligned}
F_{\text {int }}^{\infty} & =\frac{-8 \pi R^{8} w^{2} e^{-(d-2 R)}}{3 d\left[R^{3}(w+1)+R^{2}(3 w+4)+3 R(w+3)+9\right]^{2}} \\
& =-\frac{8 \pi}{3} \mathcal{A}^{2} \frac{e^{-(d-2 R)}}{d}
\end{aligned}
$$

is the asymptotic interaction free-energy. It is always attractive and has the form of a Yukawa potential 29]. In Subsection V C1 using a numerical calculation, we shall show that this asymptotic expression is quite good even up to separations of the order of $\xi$ or less.

\section{Analysis of the effective Yukawa potential}

There exists an instructive geometric view on the origin of the attractive part of the two-particle interaction mediated by the surface-induced paranematic order. It reproduces the form of the Yukawa potential of Eq. (40) under the assumption of large particles $(R \gg 1)$ and was presented by one of the authors in Ref. [17]. The basic idea is that the overlapping of the paranematic coronas of the two particles reduces the volume of the energetically unfavorable liquid crystal ordering, as illustrated in 


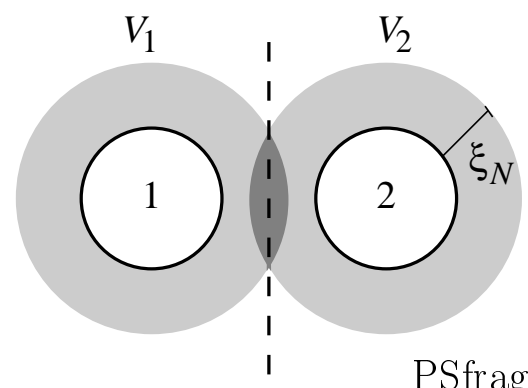

FIG. 3: The attractive paranematic interaction between the particles comes from the overlapping of the two paranematic coronas of thickness $\xi$. In the geometric view, the interaction energy is equal to the negative of the free-energy of the excess orientational order in the dark shaded region [for an exact definition see Eq. (41)]. The half spaces $V_{1}$ and $V_{2}$ are defined by the midplane of particle 1 and 2 .

Fig. 3. and therefore the total free-energy. The interaction energy is the free-energy of the removed orientational order. If we denote by $Q_{i j}^{(n)}(\boldsymbol{r})$ the single-particle orderparameter field centered on particle $n$ and switch to the representation by the components $q_{i}^{(n)}(i=0, \ldots, 4)$ [see Eq. (17)], the interaction energy is calculated as

$$
F_{\mathrm{int}}=-2 \sum_{i=0}^{4} c_{i}\left\{\int_{V_{2}}\left[\left(q_{i}^{(1)}\right)^{2}+\left(\nabla q_{i}^{(1)}\right)^{2}\right] d^{3} r\right\},
$$

where $V_{2}$ denotes the half space of particle 2 (see Fig. (3). This definition leads to the Yukawa potential of Eq. (40) in the limit $R \gg 1$, however with half the strength. Its advantage lies in its semiquantitative agreement with Eq. (40) and its simplicity. Using a type of Voronoi cell construction, it is extensible to multibody interactions which are important in the study of particle aggregation and the formation of ordered crystalline structures.

It was also demonstrated in Ref. 17] that the Yukawa potential of Eq. (40) can exactly be derived by approximating the tensorial order-parameter through a linear superposition of the two single-particle solutions centered on particle 1 and 2

$$
Q_{i j}(\boldsymbol{r})=Q_{i j}^{(1)}(\boldsymbol{r})+Q_{i j}^{(2)}(\boldsymbol{r}) .
$$

This approach is in full analogy to the treatment of the two-particle potential in electrostatically stabilized colloids based on the Debye-Hückel approximation.

\section{Derjaguin approximation}

The force between two interacting spheres is often calculated in terms of the interaction energy per unit surface $\mathcal{E}(s)$ between two parallel plates a distance $s$ apart, using the so-called Derjaguin approximation [14]. For two equal spheres of radii $R$ a distance $s$ apart, the force in the Derjaguin approximation is simply $\mathcal{F}_{D}(s)=$

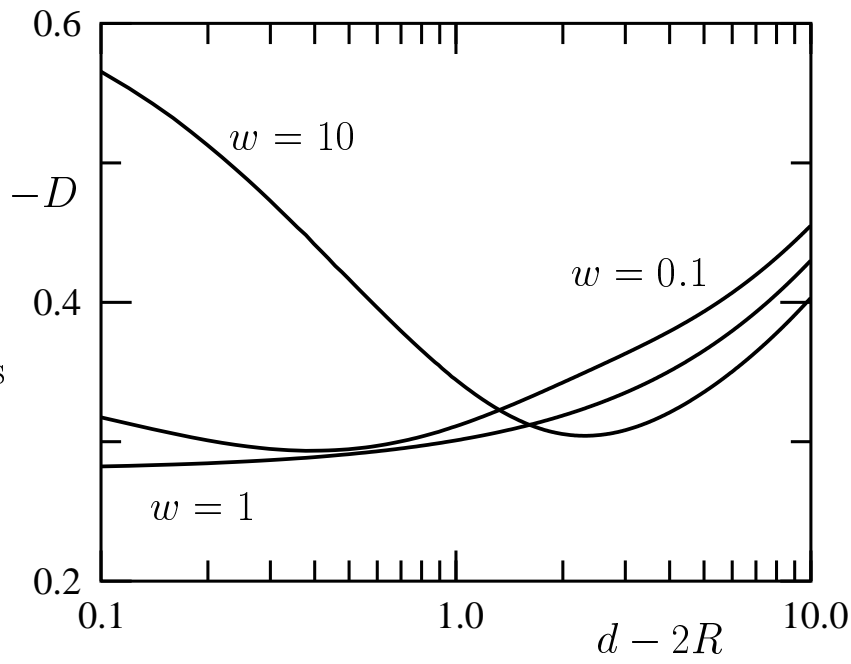

FIG. 4: Relative error $D$ of the force in the Derjaguin approximation with respect to the exact numerical one, calculated according to the procedure outlined in Section $\nabla \mathrm{C}$ The error is plotted as a function of the distance to contact $s=d-2 R$ for a particle of reduced radius $R=20$ and for various values of the reduced anchoring $w$. Contrary to what expected, the approximation does not improve at short distances.

$\pi R \mathcal{E}(s)$ 14]. Such an approximation is valid if the interactions are additive and if the radii of the particles are large with respect to both the range of the interaction and the minimum separation distance $s$ between the particles. Although in our case the interaction energy is not pairwise-additive, let us examine whether there exists a regime in which the Derjaguin approximation holds.

The interaction energy between two parallel plates, imposing homeotropic boundary conditions, immersed in a nematic liquid crystal was calculated in 12]. For weak induced paranematic order in the isotropic phase, we can use our quadratic free-energy (3). The resulting orderparameter is everywhere uniaxial, with a nematic director everywhere orthogonal to the surfaces. In our reduced units, a straightforward calculation gives the exact interaction energy per unit surface

$$
\mathcal{E}(s)=-\frac{4 w^{2}}{3(1+w)\left[e^{s}(1+w)+w-1\right]},
$$

that is everywhere attractive. The Derjaguin approximation gives the interaction force

$$
\mathcal{F}_{D}(s)=-\frac{4 \pi R w^{2}}{3(1+w)\left[e^{s}(1+w)+w-1\right]} .
$$

To check the Derjaguin approximation, we need to compare the forces and not the interaction energy. Indeed, since the Derjaguin approximation does not hold for separations large with respect to the radii of the particle, we cannot fix the correct zero level for the interaction energy. We calculate the exact force $\mathcal{F}=-\partial F_{\text {int }} / \partial d$ by numerically evaluating the interaction energy $F_{\text {int }}$, as will 


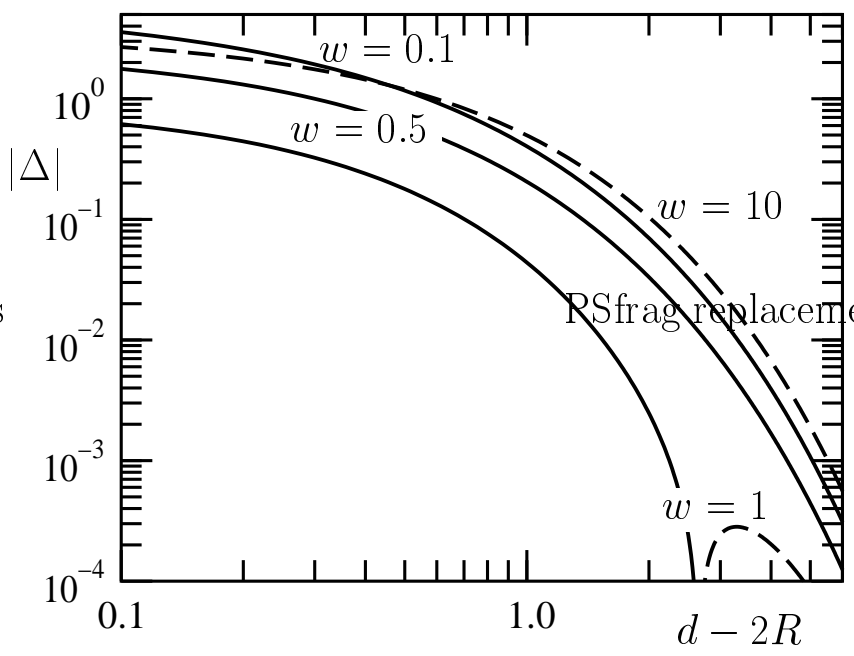

FIG. 5: Absolute value of the relative error $\Delta$ of the asymptotic interaction energy as a function of the distance to contact $d-2 R$ for $R=2$ and various values of the reduced anchoring $w$. The solid lines indicate that $\Delta>0$, the dashed lines that $\Delta<0$.

be explained in Sec. $\mathrm{VC}$ In Fig. 4 we plot the relative error $D$, defined through $\mathcal{F}=\mathcal{F}_{D}(1+D)$. As it is seen, the Derjaguin approximation is rather crude. Moreover, it does not improve as the separation distance $s$ tends to zero, as is normally expected. This is most probably due to the non pairwise-additive character of the force. The error is actually lowest in the intermediate range $1 \ll s \ll R$. Indeed, in the limit $s \gg 1$ the Derjaguin approximation (44) becomes

$$
\mathcal{F}_{D}(s) \simeq-\frac{4 \pi R w^{2} e^{-s}}{3(1+w)^{2}},
$$

which coincides, in the limit $R \gg 1$, with the exact asymptotic force $-\partial F_{\text {int }}^{\infty} / \partial d$ obtained from the asymptotic interaction energy (40), with $s=d-2 R$. Care should therefore be taken in using the Derjaguin approximation for distances to contact comparable with the nematic coherence length $\xi[13]$.

\section{Numerical results}

When the two colloidal particles are an arbitrary distance $d$ apart, we determine numerically the equilibrium order-parameter texture. To this aim, we truncate the expansions (26) at some order $\ell=\ell_{\max }$. Then, with the aid of the coordinate transformation (28) and of the orthogonality relations $\mathrm{A3}$, we project numerically the boundary conditions (27) onto the Legendre functions of the first kind $P_{\ell}{ }^{m}\left(\theta_{1}\right)$, with $m=2$ for the function $\alpha(r, \theta), m=1$ for $\beta(r, \theta)$ and $m=0$ for $\gamma(r, \theta)$, and $\ell=m, \ldots, \ell_{\max }$. This gives three decoupled sets of linear equations that we solve numerically to determine the unknown coefficients $\alpha_{\ell}$ (with $\left.\ell=2, \ldots, \ell_{\max }\right), \beta_{\ell}$

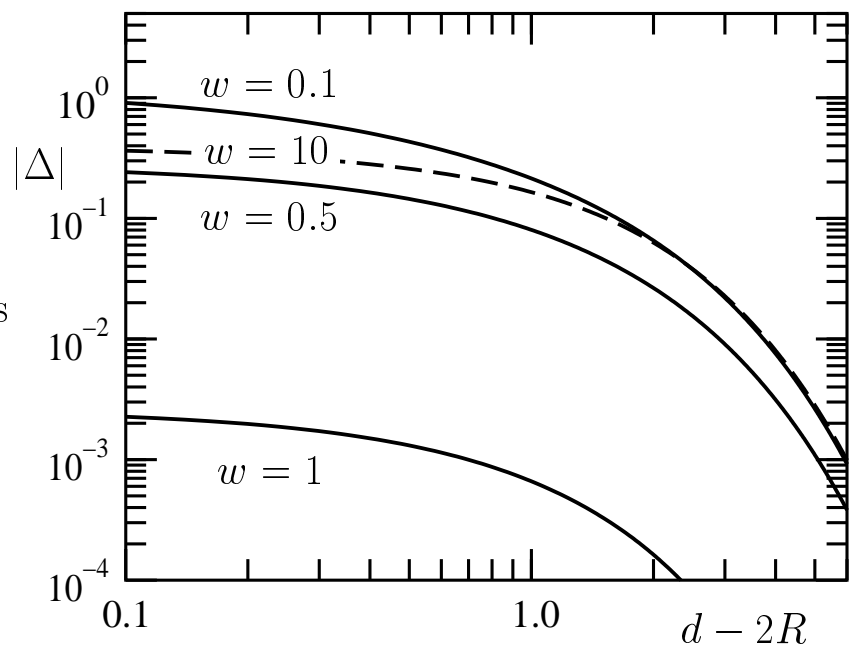

FIG. 6: As Fig. 5 but for spheres of radius $R=20$.

(with $\left.\ell=1, \ldots, \ell_{\max }\right)$, and $\gamma_{\ell}\left(\right.$ with $\left.\ell=0, \ldots, \ell_{\max }\right)$. Finally, we vary the order $\ell_{\max }$ of the expansions to obtain a given accuracy. Typically, only a few multipoles $\left(\ell_{\max } \simeq 10 \div 20\right)$ are needed to reach relative errors of the order of $10^{-3}$ or less.

\section{Interaction energy}

We determine the exact interaction energy of the colloidal particles by inserting the numerically determined expansions (26) in the equilibrium free-energy (13). The behavior of the exact interaction energy was studied in some detail in 16]. Here we concentrate on its comparison with the asymptotic expression (40), to validate the latter. We set

$$
F_{\text {int }}=F_{\text {int }}^{\infty}(1+\Delta)
$$

where $\Delta$ measures the relative error from the asymptotic solution. In Fig. [ 5 we show the behavior of $\Delta$ as a function of the distance to contact $d-2 R$ for small particles $(R=2)$ and various anchoring strength $w$. As it is seen, the asymptotic approximation is quite good up to distances to contact of the order of $\xi$. For low anchoring strength, $w<1$, the actual interaction energy is larger than the asymptotic value. For high anchoring strength, $w>1$, the actual interaction is smaller. The best agreement is for anchoring strength of the order of $w=1$, for which the relative error increases up to 0.6 at particle contact.

For relatively large particles $(R=20)$, the relative error $\Delta$ becomes even smaller, as shown in Fig. [6 It is remarkable that around $w=1$ the asymptotic approximation gives extremely good results up to contact for larger spheres. 
According to (25) and (23), the eigenvalues associated with the eigenvectors (21) of the order-parameter tensor

$$
\begin{aligned}
& \frac{\lambda_{1}(r, \theta)}{S_{0}}=\frac{\alpha(r, \theta)+\gamma(r, \theta)}{4}+\sqrt{\left[\frac{\alpha(r, \theta)-3 \gamma(r, \theta)}{4}\right]^{2}+\beta^{2}(r, \theta),} \\
& \frac{\lambda_{2}(r, \theta)}{S_{0}}=\frac{\alpha(r, \theta)+\gamma(r, \theta)}{4}-\sqrt{\left[\frac{\alpha(r, \theta)-3 \gamma(r, \theta)}{4}\right]^{2}+\beta^{2}(r, \theta),} \\
& \frac{\lambda_{3}(r, \theta)}{S_{0}}=-\frac{\alpha(r, \theta)+\gamma(r, \theta)}{2},
\end{aligned}
$$

while the angle $\Theta(r, \theta)$ that the eigenvector $\mathbf{e}^{(1)}$ makes with respect to the $z$-axis is

$$
\Theta(r, \theta)=\tan ^{-1}\left[\frac{\alpha(r, \theta)-3 \gamma(r, \theta)+\sqrt{[\alpha(r, \theta)-3 \gamma(r, \theta)]^{2}+16 \beta^{2}(r, \theta)}}{4 \beta(r, \theta)}\right] .
$$

Far from the particles, or for an isolated particle, the texture is uniaxial, with $\lambda_{2}=\lambda_{3}$. The paranematic director $\mathbf{n}$ thus coincides with the eigenvector $\mathbf{e}^{(1)}$ [see Eq. [21a] associated with $\lambda_{1}$, which is the eigenvalue of largest absolute value. Therefore, by continuity, we choose as paranematic director $\mathbf{n}$ the direction $\mathbf{e}^{(1)}$. In general, however, the tensor order-parameter Q is biaxial and is characterized by the uniaxial scalar orderparameter $S$ in the direction of the paranematic director and by the biaxiality $B$ in the plane orthogonal to it. They are defined by setting in the diagonal frame $\left(\mathbf{e}^{(1)}, \mathbf{e}^{(2)}, \mathbf{e}^{(3)}\right)$, in which $Q=\operatorname{diag}\left(\lambda_{1}, \lambda_{2}, \lambda_{3}\right)$, $Q=\operatorname{diag}(2 / 3 S,-1 / 3 S+B,-1 / 3 S-B)$ [22]. Therefore:

$$
\begin{aligned}
S(r, \theta) & =\frac{3}{2} \lambda_{1}(r, \theta), \\
B(r, \theta) & =\frac{1}{2}\left[\lambda_{1}(r, \theta)+2 \lambda_{2}(r, \theta)\right] .
\end{aligned}
$$

In Fig. $\mathbf{7}$ we have plotted the contour lines of the scalar order-parameters $S$ (top) and $B$ (middle), and the field-lines of the paranematic director $\mathbf{n}$ (bottom) for small spheres, i.e., $R=2$. In Fig. 8 we have plotted the same parameters for large spheres, i.e., $R=20$. When the two spheres are close to each other, the scalar order-parameter makes a diffuse halo around the two spheres for the small spheres, whereas it is significantly nonzero only in the gap between the spheres for the large ones. The biaxiality is maximum in the vicinity of a ring which defines a defect, of topological charge $-1 / 2$, of the paranematic director $\mathbf{n}$. The defect location corresponds to the condition $S=B$, i.e., $\lambda_{1}=\lambda_{2}$ [see Eqs. (49)]: the two orthogonal direction $\mathbf{e}^{(1)}$ and $\mathbf{e}^{(2)}$ in the symmetry plane $\Pi_{P}$ (the plane of Fig. 2 containing the $z$-axis and the generic point $P$ ) become equivalent. Strictly speaking, at the defect location the texture becomes again uniaxial, with a paranematic director orthogonal to the $\Pi_{P}$-plane and a discotic-like order. The main difference between the small and the large spheres is that the defect is in the region where $S$ is large in the former case, whereas it is located in a region where $S$ is almost zero in the latter. Regarding the texture, one can see that the field lines are rather smoothly bent in the case of small spheres, while for large ones they start almost radially from the particles and present a kink in the mid-plane, in the region where $S$ is small.

\section{Defect position}

The defect ring is located in the plane $z=0$ of Fig. 2 On this plane, $\beta=0$ for symmetry reasons. Then, the location of the defect, which corresponds to the condition $\lambda_{1}=\lambda_{2}$, according to Eqs. (47a) and (47b) is such that $\alpha(r, \theta=\pi / 2)=3 \gamma(r, \theta=\pi / 2)$. Let us calculate asymptotically, for $d \gg 1$, the defect position. At zeroth order, the total $Q$ tensor is the superposition of the $Q$ tensors of the two isolated particles. Then, one easily finds that the defect is located at a radial distance $h=d / 2$ from the axis joining the centers of the particles. This corresponds to a defect ring having a diameter equal to the distance between the centers of the two spheres.

To verify this lowest-order prediction, in Fig. 9 we plot the ratio between the ring diameter $2 h$ and the distance between the two spheres as a function of the distance to contact $d-2 R$. As one sees, the approximate estimation 

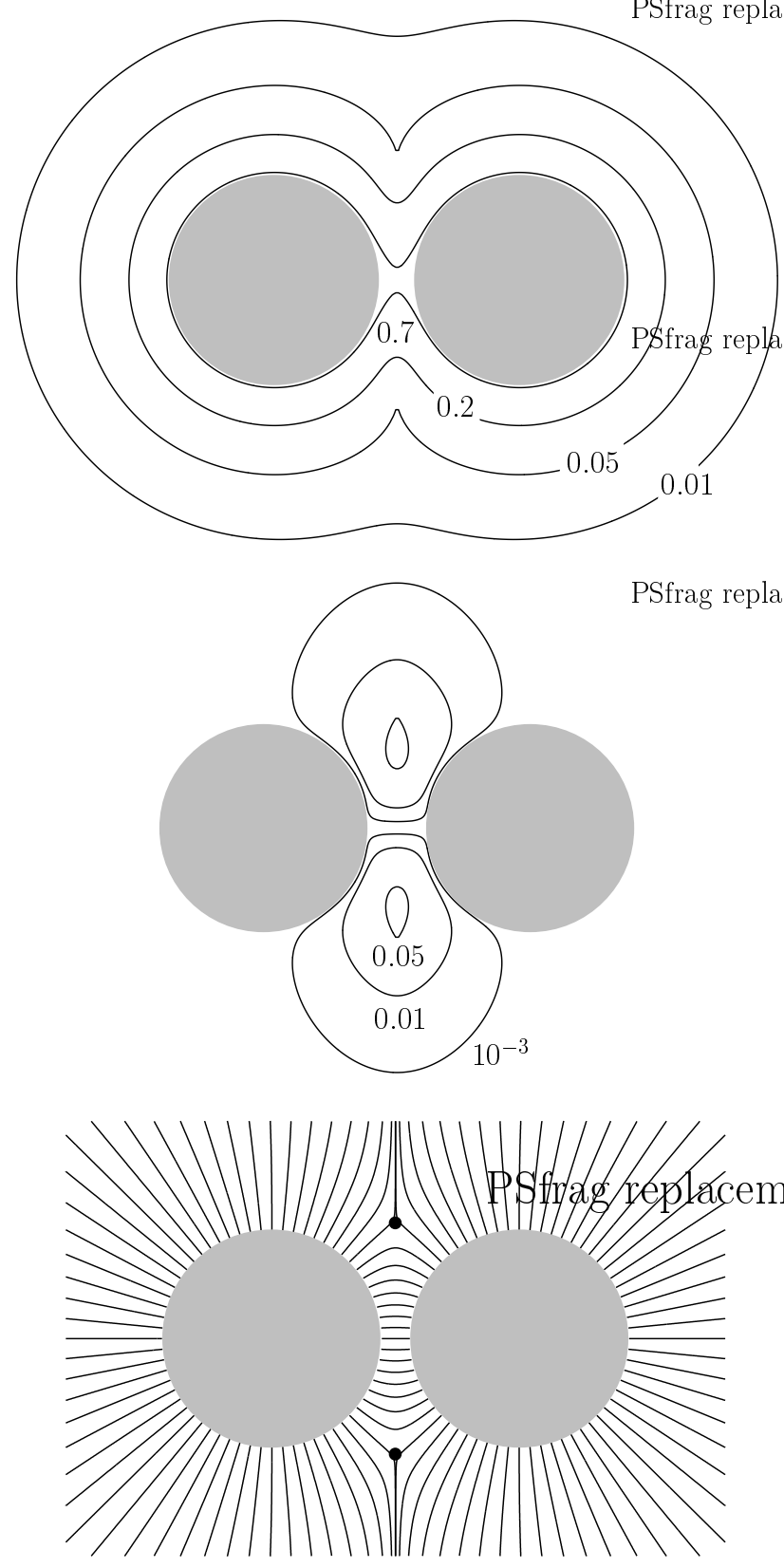

FIG. 7: Paranematic order-parameter between two spheres with reduced radius $R=2$ and reduced anchoring $w=4$. Top: contour lines of the scalar order-parameter $S$; middle: contour lines of the biaxiality parameter $B$; bottom: field lines of the paranematic director $\mathbf{n}$.

is rather good: for lower anchoring strength, the defect diameter is actually slightly larger, while for higher anchoring it is smaller. The deviation from the lowest-order solution $h=d / 2$ decreases as the spheres move further apart or become bigger. In this case, the defect ring lives in a region where the order-parameter is small.
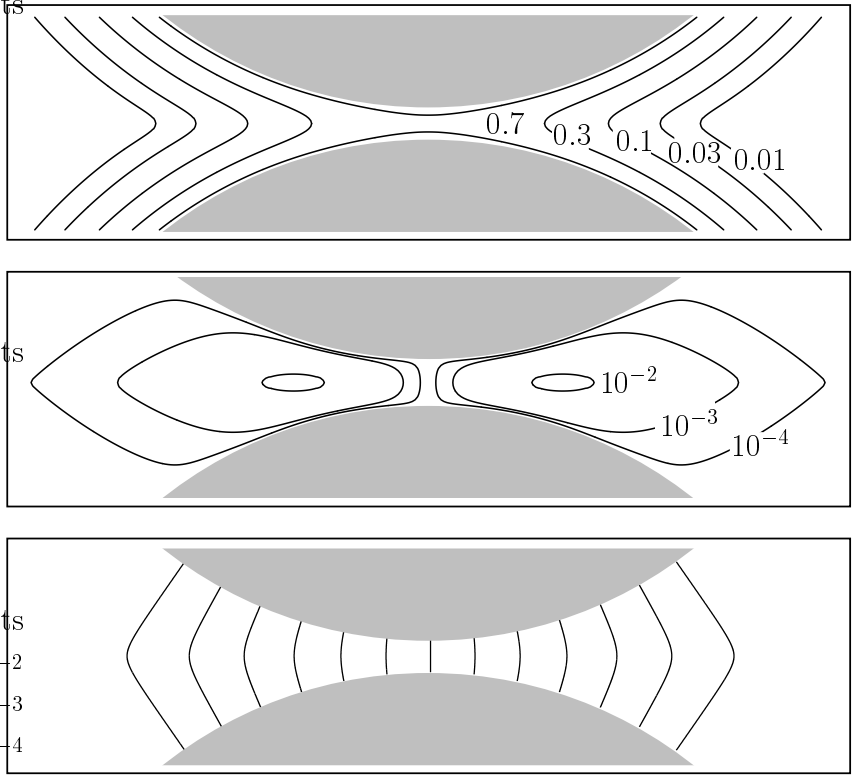

FIG. 8: Same as Fig. 7 but for $R=20$.

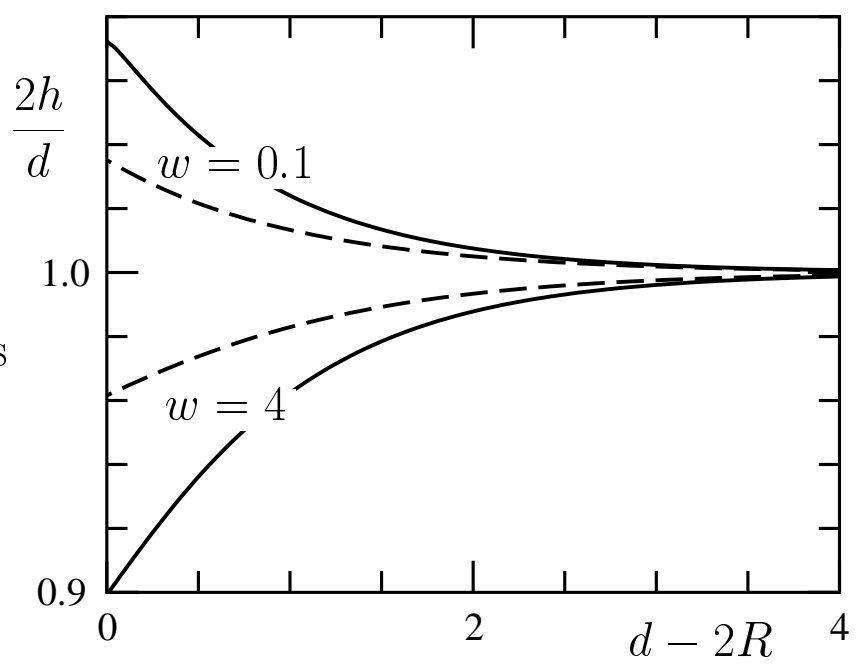

FIG. 9: Ratio between the defect ring diameter $2 h$ and the distance between the spheres $d$ as a function of the distance to contact $d-2 R$ of the spheres. Full lines: $R=2$; dashed lines: $R=5$. The two upper curves are for a normalized anchoring strength $w=0.1$, the two lower ones for $w=4$.

\section{FLOCCULATION TRANSITION}

Section $\nabla C 1$ (see also Ref. 21] ) and the Yukawa potential of Eq. (40) show that for large particles $(R \gg 1)$, the two-particle interaction mediated by the nematic wetting layer (in the following abbreviated by $U_{L C}$ ) is dominated by its strong attractive part. The consequences of this feature on the stability of a dispersion of colloidal particles were investigated in detail by Borštnik, Stark and Žumer in Ref. 20]. The main idea was to use, e.g., the screened electrostatic interaction of charged parti- 
cles to stabilize a colloidal suspension against the attractive van der Waals interaction well above the nematicisotropic phase transition. Then by lowering the temperature towards the transition temperature $T_{c}$, which increases both the strength and range of the liquid-crystal mediated interaction, a flocculation transition can be induced, i.e., the particles start to aggregate. Whereas the transition itself has not been observed so far, the attractive two-particle potential has been demonstrated in excellent experiments by Kočevar and Muševič using an atomic force microscope [30, 31].

In this article, we study again the flocculation transition for two reasons. In Ref. [20] the interaction potential $U_{L C}$ was calculated by modeling the liquid-crystalline order with the help of an ansatz function. A weak repulsive barrier in $U_{L C}$ occurred which seems to be an artifact in the construction of the ansatz function. Secondly, by using the analytic form of the Yukawa potential for $U_{L C}$, the calculation of the total interaction energy becomes much easier. Therefore, in Section VIB we shall discuss a flocculation diagram in terms of the relevant parameters of the electrostatic interaction which covers a much larger range than in Ref. [20]. We will, however, confirm the flocculation diagram of Ref. [20].

\section{A. Two-particle interactions}

In the following we consider a colloidal dispersion of particles subject to van der Waals $\left(U_{W}\right)$, electrostatic $\left(U_{E}\right)$, and liquid crystal induced $\left(U_{L C}\right)$ interactions, where $U_{W}$ and $U_{E}$ are taken according to the DLVO theory 32,33 . In a first approach, we assume that the surface induced nematic order has no effect on $U_{W}$ and $U_{E}$, so that the total two-particle potential can be written as $U_{\text {tot }}=U_{L C}+U_{W}+U_{E}$.

To be concrete, we consider silica particles of radius $R=250 \mathrm{~nm}$ dispersed in the liquid crystal 4- $n$-octyl-4'cyanobiphenyl (8CB). The van der Waals interaction between identical particles is always attractive. It reads [34]

$$
U_{W}=-\frac{H}{6}\left[\frac{2 R^{2}}{d^{2}-4 R^{2}}+\frac{2 R^{2}}{d^{2}}+\ln \left(\frac{d^{2}-4 R^{2}}{d^{2}}\right)\right]
$$

where $d$ is the center-of-mass separation of the particles and $H=1.1 k_{\mathrm{B}} T$ is the Hamaker constant for silica particles suspended in the compound 8CB [20]. Strictly speaking, via retardation effects, the Hamaker constant also depends on the separation $d$ [34]. However, in our problem this dependence is not crucial [20]. Note that in the following we do not use reduced units: all physical quantities keep their dimensions.

We stabilize the colloidal dispersion by electrostatic repulsion. The particles possess an electrical surface charge density $q_{s}$, and monovalent salt of concentration $n_{p}$ is added to the solvent. This results in the two-particle potential 34$]$

$$
U_{E}=-\frac{8 \pi^{2}}{\varepsilon_{2}} \frac{R q_{s}^{2}}{\kappa^{2}} \ln \left(1-e^{-\kappa(d-2 R)}\right) .
$$

The range of the interaction is determined by the Debye length $\kappa^{-1}=\left[\varepsilon_{2} k_{\mathrm{B}} T /\left(8 \pi e^{2} n_{p}\right)\right]^{1 / 2}$, where $\varepsilon_{2}$ denotes the dielectric constant of the solvent and $e$ is the fundamental charge. Note that Eq. (51) is derived in the Derjaguin approximation under the assumption $d-2 R, \kappa^{-1} \ll R$. For realistic values of $\kappa^{-1}$ and $q_{s}$, see Ref. [20] and [31].

To include the liquid-crystal mediated interaction, we use the Yukawa potential of Eq. (40). The latter, restoring to all the physical quantities their dimensions, is given explicitly by

$$
U_{L C}=-\frac{8 \pi}{3} L \xi^{2}\left(S_{0} \mathcal{A}\right)^{2} \frac{e^{-(d-2 R) / \xi}}{d},
$$

where the amplitude $\mathcal{A}$ must be calculated according to Eq. (18), with $R$ substituted by $R / \xi$ and with $w=$ $W /\left[L \alpha\left(T-T^{*}\right)\right]^{1 / 2}$ the reduced surface coupling constant. We recall also that the nematic coherence length is given by $\xi=\left[L /\left(\alpha\left(T-T^{*}\right)\right)\right]^{1 / 2}$. In Section $\nabla C$ it was shown that for large particle radii the Yukawa potential agrees well with the numerical results down to a distance to contact of the order of $\xi$. Even for smaller distances it gives a good approximation to the interaction energy. We use Eq. (52) to obtain an estimate for the strength of the Yukawa potential in the case $R \gg \xi$ :

$$
\left|U_{L C}(d=2 R)\right| \simeq \frac{4 \pi}{3} L R S_{0}^{2}\left(\frac{w}{w+1}\right)^{2} .
$$

The rational function in the reduced surface coupling constant $w$ is monotonously growing like $w^{2}$ for small $w$ and approaching one at large $w$. Therefore, we find that the strength of the Yukawa potential increases, like its range $\xi$, when the temperature is lowered towards the nematic-isotropic phase transition at $T_{c}$. For example, for the material parameters chosen immediately below, we find that the strength increases by a factor of six when cooling down from $T_{c}+10 \mathrm{~K}$ to $T_{c}$. When discussing the effect of the interaction $U_{L C}$, we shall use the parameters of $8 \mathrm{CB}\left[\alpha=0.12 \times 10^{7} \mathrm{erg} /\left(\mathrm{cm}^{3} \mathrm{~K}\right), L=1.8 \times 10^{-6} \mathrm{dyn}\right.$, $T_{c}-T^{*}=1.3 \mathrm{~K}$, and $\left.T_{c}=314.8 \mathrm{~K}\right]$, which give a coherence length $\xi\left(T_{c}\right)=10.74 \mathrm{~nm}$ at the phase transition. Furthermore, we choose $W=1.25 \mathrm{erg} / \mathrm{cm}^{2}$ and $S_{0}=$ 0.45, in accord with Ref. [20]. Finally, all interactions in the next subsection are referred to the thermal energy $k_{\mathrm{B}} T$ at room temperature, and, as already mentioned, the particle radius is $250 \mathrm{~nm}$.

\section{B. Flocculation diagram}

When discussing the total interaction energy for varying parameters, we will find different shapes of the twoparticle potential which affect the flocculation transition. 


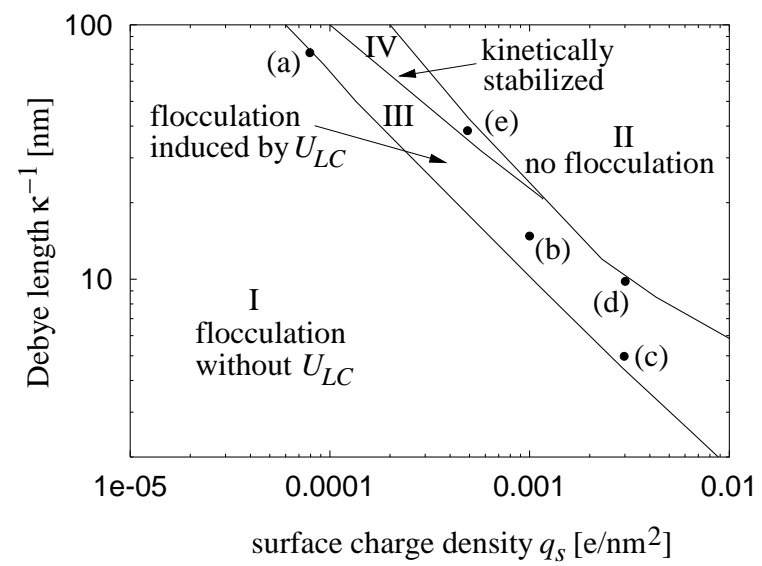

FIG. 10: Flocculation diagram in terms of the tunable parameters of the electrostatic interaction, i.e., the surface charge density $q_{s}$ and the Debye length $\kappa^{-1}$. The four different regions I-IV characterize how a colloidal dispersion of charged particles reacts on $U_{L C}$. The points (a)-(e) refer to the diagrams in Figs. 11 and 12

Particles start to aggregate when the interaction potential exhibits a minimum $U_{\min }<0$ at finite distances. If the potential minimum is shallow, i.e., just several $k_{\mathrm{B}} T$ deep, a particle doublet will break up again, and a phase equilibrium of an aggregated and dispersed state occurs [34]. The higher potential energy in the dispersed state is compensated by the larger entropy. On the other hand, when $\left|U_{\min }\right|$ tends to $10 k_{\mathrm{B}} T$ or even higher values, strong attraction occurs that leads to a non-equilibrium phase with all the particles aggregated. This corresponds to the flocculation transition that we aim to investigate. As a further feature of our interaction potentials, we will also encounter potential barriers which slow down the flocculation or even "kinetically stabilize" the dispersion.

Obviously, the observation of a flocculation transition is a matter of time scale on which the experiments take place. The theory of aggregation kinetics determines the characteristic time $\tau$ for the formation of a particle doublet as [34, 35]

$$
\tau=\frac{1}{\phi} \frac{R^{2}}{6 D_{0}} I
$$

where the factor $I$ is given by

$$
I=2 R \int_{s_{\min }}^{\infty} \frac{D_{0}}{D(s)} \frac{\exp \left(U_{\mathrm{tot}} / k_{\mathrm{B}} T\right)}{(2 R+s)^{2}} d s,
$$

which contains the ratio

$$
\frac{D(s)}{D_{0}}=\left\{\begin{array}{ll}
2 s / R & \text { for } s \ll R \\
1 & \text { for } s \gg R
\end{array} .\right.
$$

In Eq. (54), $\phi$ is the volume fraction of the particles in the solvent, and $D_{0}=k_{B} T /(6 \pi \eta R)$ is the single particle diffusion coefficient depending on the solvent viscosity $\eta$.
For particles that stick together when they meet but otherwise do not interact $(I=1), \tau$ is given by the time an independent particle needs to diffuse a distance $R$ times the inverse volume fraction $\phi$. Clearly, in a very dilute dispersion ( $\phi$ small), particles hardly come close to each other and doublet formation is very rare. The factor $I$, where $s=d-2 R$ denotes the distance to contact, incorporates influences from the two-particle interaction; in the presence of a potential barrier, $\tau$ increases considerably even for moderate $\phi$. The ratio $D_{0} / D(s)$ takes into account corrections of the diffusion constant due to hydrodynamic interactions. Note that $s_{\min }$ generally means $s=0$, i.e., the particles stick together at direct contact. If, however, for very small particle separations a strong repulsion sets in, $s_{\min }$ is taken as the location of the potential minimum which is responsible for the formation of the particle doublet.

In the following, we assume a volume fraction $\phi=0.1$ so that for a typical shear viscosity of $0.4 \mathrm{P}$ we arrive at $\tau / I=5 \mathrm{~s}$. We say that a dispersion is kinetically stabilized by a potential barrier if $\tau>1 \mathrm{~h}$, which corresponds to $I=720$. With the help of the algebraic program Maple, we have numerically calculated the factor $I$ for our potential $U_{\text {tot }}$ to check when such a kinetic stabilization in the flocculation diagram, to be discussed below, sets in. In this way, we have determined the transition lines between regions I/III and III/IV in Fig. 10. To gain further insight into the factor $I$, we perform a saddle-point approximation. We replace $U_{\text {tot }}$ by its harmonic approximation around the maximum $U_{\max }$ of the potential barrier. Then we can evaluate the integral in Eq. (55) when we approximate $s$ by $s_{\max }$ otherwise and choose $s_{\min }=0$. From the magnitude of the curvature $c_{0}=-\partial^{2} U_{\text {tot }} /\left.\partial s^{2}\right|_{s_{\max }}$, we deduce a characteristic length $\xi=\left(2 k_{\mathrm{B}} T / c_{0}\right)^{1 / 2}$ and make the approximation of replacing $s_{\max }$ by $\xi$ which finally gives the estimate $I=\exp \left(U_{\max } / k_{\mathrm{B}} T\right) / 2$. From our criterion for kinetic stabilization, $I=720$, we find $U_{\max }=7.3 k_{\mathrm{B}} T$. Surprisingly, in most cases the exact calculation of the integral in Eq. (55) gave potential barriers with $U_{\max }$ between 7 and $8 k_{\mathrm{B}} T$. This demonstrates that the Boltzmann factor is the determining quantity in $I$ and therefore in $\tau$.

We will also encounter two-particle interactions where the particle doublet settles into a potential minimum without traversing a potential barrier. Here the question arises how stable the doublet is. Unfortunately, the familiar Kramers rate [36] is not applicable, since it involves the curvature of the potential maximum over which a particle escapes. In our case this potential maximum is located at infinity $\left(U_{\text {tot }}=0\right)$. It has zero curvature, which gives an infinite escape time. The theory for our problem formulates the escape time as a double integral over the particle separation [37], that cannot be calculated straightforwardly. However, we can give an upper bound for the escape time 37], defined as the time $t_{\text {diff }}$ that a particle needs to diffuse a distance $R$ when leaving 

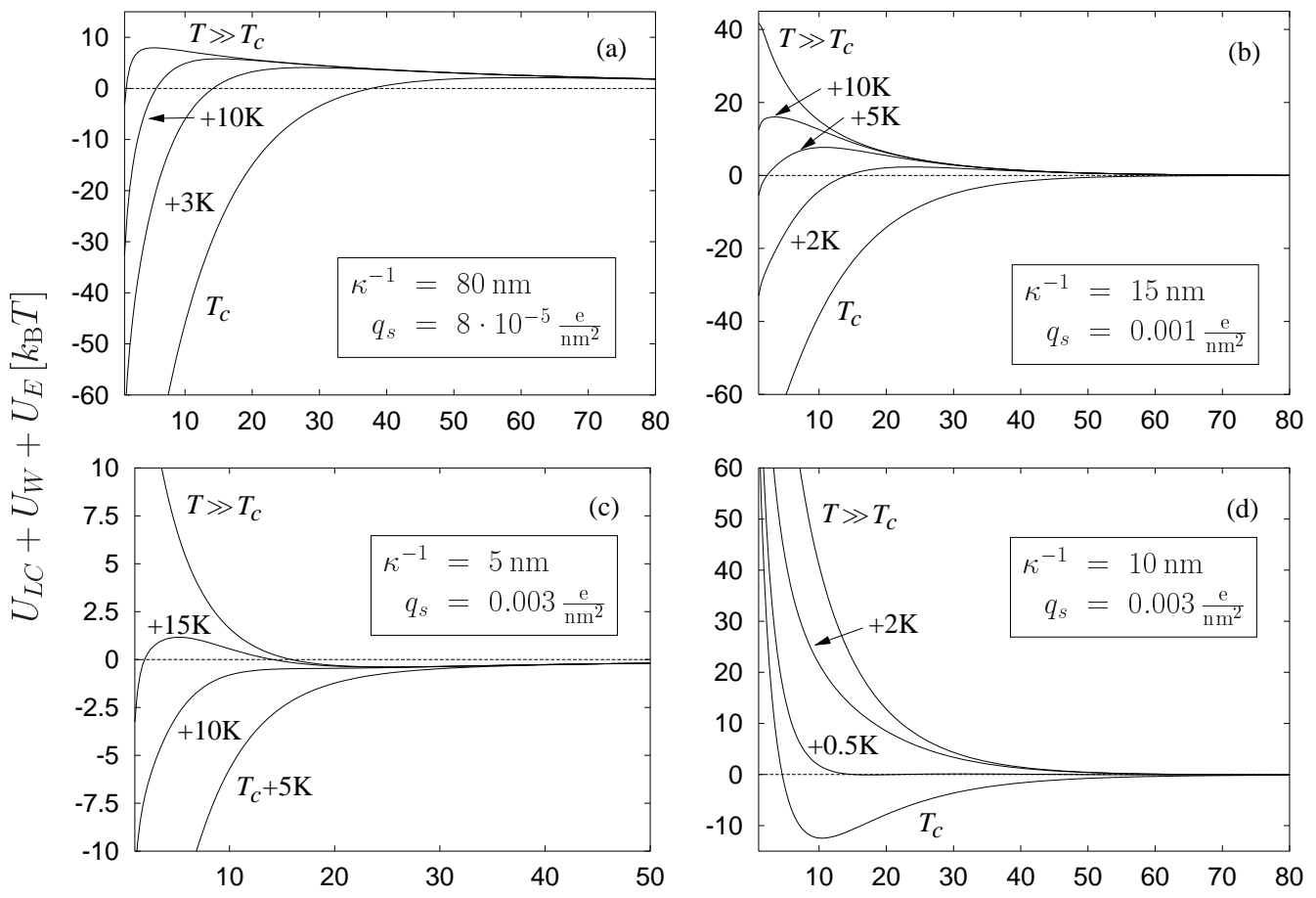

distance to contact $d-2 R\lceil\mathrm{~nm}\rceil$

FIG. 11: Total two-particle potential in units of $k_{\mathrm{B}} T$ as a function of distance to contact $d-2 R$. The potentials are shown for different parameters of the electrostatic interaction, as indicated in the inset, and at various temperatures relative to $T_{c}$. The labels (a)-(d) refer to a location in the flocculation diagram of Fig. 10

a potential well of depth $U_{0}$

$$
t_{\text {diff }}=\frac{R^{2}}{6 D_{0} \exp \left(-U_{0} / k_{\mathrm{B}} T\right)} .
$$

Again, we consider a particle doublet as stable when $t_{\text {diff }}$ exceeds $1 \mathrm{~h}$, which for the parameters already introduced results in a potential depth of $U_{0}=9 k_{\mathrm{B}} T$. Compared to the free diffusion time $R^{2} /\left(6 D_{0}\right)=0.5 \mathrm{~s}$, it enhances $t_{\text {diff }}$ by a factor of 7200 . We used this criterion to establish the boundary line between region II and III in Fig. 10]

In the following, we discuss in detail the flocculation diagram of Fig. 10 (calculated for a particle radius $R$ of $250 \mathrm{~nm}$ ). It is plotted in terms of the tunable parameters of the electrostatic interaction, i.e., the surface charge density $q_{s}$ and the Debye length $\kappa^{-1}$. We distinguish four regions: in region I the particles are completely aggregated without $U_{L C}$ since the electrostatic interaction is not sufficient to stabilize the dispersion against the van der Waals force. For large surface charge density and interaction range, as in region II, the dispersion is completely stabilized even if $U_{L C}$ is turned on. A potential minimum at finite distances does not occur, or it is not deep enough, according to our discussion above, to trigger flocculation. Both regions are separated by areas III and IV. In region III flocculation is induced by lowering the temperature towards $T_{c}$. Different types of interaction potentials occur which are illustrated in Fig. 11] for various locations in the flocculation diagram of Fig. 10 labeled by (a) to (d). In diagram (a), the graph for $T \gg T_{c}$ exhibits the usual "primary minimum" at short distances due to the van der Waals interaction which is followed by a "primary potential barrier" for increasing separations well known in colloids science. The electrostatic repulsion is just strong enough to stabilize the colloidal suspension kinetically. Lowering temperature reduces the barrier and induces flocculation. Moving down the diagonal from left to right in the flocculation diagram, we arrive at location (b). In the corresponding Fig. 11(b), the primary minimum at $T \gg T_{c}$ is no longer visible due to the increase in the surface charge density. To induce flocculation, one has to cool the dispersion closer to $T_{c}$. Moving further down the diagonal, a shallow potential minimum at around $26 \mathrm{~nm}$ appears at $T \gg T_{c}$, as illustrated in Fig. 11](c), which leads to a slight phase coexistence. However, a clear non-reversible aggregation of the particles will be induced when the temperature is decreased. A new feature occurs in diagram[11(d), which is located close to the transition line to the stabilized dispersion. Whereas in the previous cases the flocculation transition sets in gradually with decreasing temperature, here a sudden transition is observable within a few tenths of Kelvin. Crossing the transition line from location (d), the minimum at $T=T_{c}$ becomes more shallow and ultimately vanishes. Region IV in the flocculation diagram 


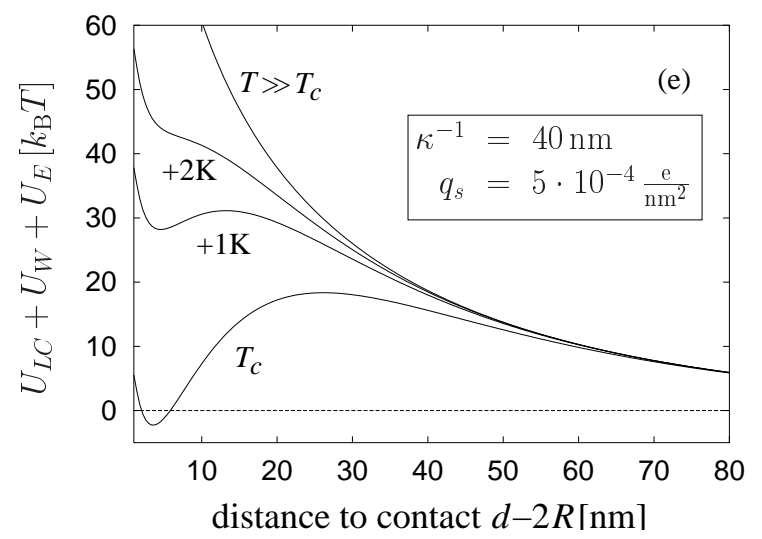

FIG. 12: Total two-particle potential in units of $k_{\mathrm{B}} T$ as a function of distance to contact $d-2 R$. The potential is shown at various temperatures relative to $T_{c}$. The parameters of the electrostatic interaction is shown in the inset. The label (e) refers to a location in the flocculation diagram of Fig. 10.

of Fig.10 identifies a kinetically stabilized dispersion. As illustrated by Fig. 12 at $T=T_{c}$ a minimum in the total interaction energy is separated from large distances by a potential barrier which prevents the formation of stable particle doublets. The interaction becomes totally repulsive when moving into region II.

\section{CONCLUSIONS}

In this paper, following previous works 15, 16, 17, 20, 21], we have reconsidered the interactions between spherical colloids wetted by a corona of paranematic order in the isotropic phase of a nematogenic material. We have calculated the contribution to the total interaction that is mediated by the paranematic order, assuming a radial anchoring of the director. Our results, based on a quadratic approximation of the free-energy density, are exact in the limit of weak induced paranematic order if the temperature is not too close to the nematic-isotropic phase transition. We have obtained analytical results in the asymptotic regime where the distance between the colloids is large with respect to the coherence length $\xi$ of the nematic order, and numerical results for any separation. For large separations, the interaction follows the Yukawa-like potential given by Eq. (40), which can be explained on simple grounds. Our numerical results show that, for colloids large compared to $\xi$, this simple potential is actually a very good approximation even up to separations of the order of $\xi$. We obtain attractive interactions for colloids much larger than $\xi$ and the possibility of short-range repulsions for colloids of size comparable to $\xi$. We have discussed the paranematic texture between two colloidal particles, showing that a defect of the paranematic director, in the form of a ring of topological charge $-1 / 2$ appears in the midplane of the two particles. The diameter of the ring is approximately equal to the distance between the centers of the two particles, which is easily explained by supposing that at lowest order the total paranematic tensor is the superposition of the paranematic tensors for the two isolated particles. The ring is surrounded by a sheath of biaxial order.

Finally, using the Yukawa-like expression (40) for the paranematic interaction, we have examined the stability of a colloidal suspension by considering the interplay of the paranematic interaction with the standard DVLO interactions, i.e., van der Waals attractions and doublelayer electrical repulsions. We have found that the stability of a colloidal suspension can be significantly affected by the paranematic interaction: the latter can either trigger flocculation or kinetically stabilize the suspension depending on the vicinity of the isotropic-nematic transition of the nematogenic solvent and on the DLVO parameters. These properties could be tested experimentally.

\section{APPENDIX A: DEFINITION OF THE SPHERICAL HARMONICS}

Among the various possible definitions of the spherical harmonics, we use the form

$$
Y_{\ell m}(\theta, \phi)=\sqrt{\frac{2 \ell+1}{4 \pi} \frac{(\ell-m) !}{(\ell+m) !}} P_{\ell}{ }^{m}(\cos \theta) e^{i m \phi},
$$

where the $P_{\ell}^{m}(\cos \theta)$ are associated Legendre functions of the first kind:

$$
P_{\ell}^{m}(t)=\frac{(-1)^{\ell+m}}{2^{\ell} \ell !}\left(1-t^{2}\right)^{m / 2} \frac{d^{\ell+m}}{d t^{\ell+m}}\left(1-t^{2}\right)^{\ell} .
$$

The Legendre functions obey the orthogonality relations

$$
\int_{-1}^{1} P_{\ell}^{m}(t) P_{\ell^{\prime}}{ }^{m}(t) d t=\frac{(\ell+m) !}{(\ell+1 / 2)(\ell-m) !} \delta_{\ell \ell^{\prime}}
$$

where $\delta_{\ell \ell^{\prime}}$ is the Kronecker delta. These orthogonality relations imply the orthonormality of the spherical harmonics

$$
\int Y_{\ell m}(\theta, \phi) Y_{\ell^{\prime} m^{\prime}}^{*}(\theta, \phi) \sin \theta d \theta d \phi=\delta_{\ell \ell^{\prime}} \delta_{m m^{\prime}}
$$

[1] R. J. Hunter, Foundations of Colloid Science (Clarendon Press, Oxford, 1989).
[2] E. M. Terentjev, Phys. Rev. E 51, 1330 (1995).

[3] P. Poulin, V. Cabuil, and D. A. Weitz, Phys. Rev. Lett. 
79, 4862 (1997).

[4] P. Poulin, H. Stark, T. Lubensky, and D. Weitz, Science 275, 1770 (1997).

[5] P. Poulin and D. A. Weitz, Phys. Rev. E 57, 626 (1998).

[6] H. Stark, Phys. Rep. 351, 387 (2001).

[7] P. Poulin, N. Francès, and O. Mondain-Monval, Phys. Rev. E 59, 4384 (1999).

[8] M. Zapotocky, L. Ramos, P. Poulin, and T. C. Lubensky, Science 283, 209 (1999).

[9] S. P. Meeker, W. C. K. Poon, J. Crain, and E. M. Terentjev, Phys. Rev. E 61, R6083 (2000).

[10] V. J. Anderson and E. M. Terentjev, Eur. Phys. J. E 4, 21 (2001).

[11] J. C. Loudet, P. Barois, and P. Poulin, Nature 407, 611 (2000).

[12] A. Borštnik and S. Žumer, Phys. Rev. E 56, 3021 (1997).

[13] K. Kočevar and I. Muševič, Phys. Rev. E 64, 051711 (2001).

[14] J. Israelachvili, Intermolecular and Surface Forces (Academic Press, San Diego, CA, 1992).

[15] A. Borštnik, H. Stark, and S. Žumer, Phys. Rev. E 60, 4210 (1999).

[16] P. Galatola and J.-B. Fournier, Phys. Rev. Lett. 86, 3915 (2001).

[17] H. Stark, Phys. Rev. E 66, 041705 (2002).

[18] H. Löwen, Phys. Rev. Lett. 74, 1028 (1995).

[19] C. Bauer, T. Bieker, and S. Dietrich, Phys. Rev. E 62, 5324 (2000).

[20] A. Borštnik, H. Stark, and S. Žumer, Phys. Rev. E 61, 2831 (2000).

[21] J.-B. Fournier and P. Galatola, Phys. Rev. E 65, 032702 (2002).
[22] P.-G. de Gennes and J. Prost, The Physics of Liquid Crystals (Clarendon, Oxford, 1993).

[23] S. Kralj, E. G. Virga, and S. Žumer, Phys. Rev. E 60, 1858 (1999).

[24] G. Vertogen and W. H. de Jeu, Thermotropic Liquid Crystals (Springer-Verlag, Berlin, 1988).

[25] S. Kralj and S. Žumer, Phys. Rev. A 45, 2461 (1992).

[26] P.-G. de Gennes, Mol. Cryst. Liq. Cryst. 12, 193 (1971).

[27] W. J. A. Gossens, Mol. Cryst. Liq. Cryst. 124, 305 (1985).

[28] M. Nobili and G. Durand, Phys. Rev. A 46, R6174 (1992).

[29] Y. Nambu, Quarks, Frontiers in Elementary Particle Physics (World Scientific, New York, 1985).

[30] K. Kočevar, A. Borštnik, I. Muševič, and S. Žumer, Phys. Rev. Lett. 86, 5914 (2001).

[31] K. Kočevar and I. Muševič, Phys. Rev. E 65, 030703(R) (2002).

[32] B. V. Derjaguin and L. Landau, Acta Physiochem. 14, 633 (1941).

[33] E. G. Verwey and J. T. G. Overbeek, The Theory of the Stability of Lyophilic Colloids (Elsevier, Amsterdam, 1948).

[34] W. B. Russel, D. A. Saville, and W. R. Schowalter, Colloidal Dispersions (Cambridge University Press, Cambridge, 1995).

[35] D. C. Prieve and E. Ruckenstein, J. Colloid Interface Sci. 73, 539 (1980).

[36] H. Kramers, Physica (Utrecht) 7, 284 (1940).

[37] S. Lifson and J. L. Jackson, J. Chem. Phys. 36, 2410 (1962). 